\title{
新型熊果酸苯并咪(噻)唑连苯酯合成及抗炎活性测定
}

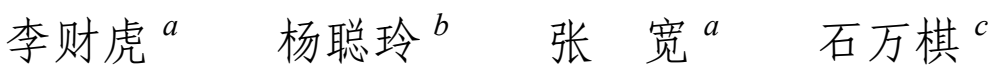 \\ 李剑忠 $b$ 李 颖*, $a$ 尹述凡*,a \\ $\left({ }^{a}\right.$ 四川大学化学学院 成都 610064) \\ ( ${ }^{b}$ 四川师范大学化学与材料科学学院 成都 610066) \\ $\left({ }^{c}\right.$ 四川国康药业有限公司 成都 610041)
}

\begin{abstract}
摘要 以熊果酸、取代苯甲醛和邻苯二胺或邻氨基苯硫酚为原料, 合成了一系列熊果酸苯并咪(噻)唑连苯酯衍生物 $\mathbf{4 a} \sim \mathbf{4 h}, 8$ 个新化合物均未见文献报道, 其结构经 ${ }^{1} \mathrm{H}$ NMR, IR 和 HRMS 加以确认，并对 $\mathbf{4 a} \sim \mathbf{4 h}$ 进行药理活性篎选，结 果表明, 化合物 $\mathbf{4 d}\left(150 \mathrm{mg} \cdot \mathrm{kg}^{-1}\right), \mathbf{4 g}\left(150 \mathrm{mg} \cdot \mathrm{kg}^{-1}\right)$ 等具有良好抗炎活性.

关键词 熊果酸; 苯并咪唑; 苯并噻唑; 抗炎
\end{abstract}

\section{Synthesis and Anti-inflammatory Activity of Novel Benzimida- zole (Benzothiazole) Phenylursolate}

\author{
Li, Caihu $^{a} \quad$ Yang, Congling $^{b} \quad$ Zhang, Kuan $^{a} \quad$ Shi, Wanqi ${ }^{c}$ \\ Li, Jianzhong $^{b} \quad$ Li, Ying*,a Yin, Shufan*,a \\ ( ${ }^{a}$ Faculty of Chemistry Sichuan University, Chengdu 610064) \\ ( ${ }^{b}$ College of Chemical and Material Science, Sichuan Normal University, Chengdu 610066) \\ ( ${ }^{c}$ Sichuan Guokang Pharmaceutical Co., Ltd, Chengdu 610041)
}

\begin{abstract}
A novel series of the benzimidazole or benzothiazole phenyl ester derivatives of ursolic acid $\mathbf{4 a} \sim \mathbf{4 h}$ were prepared via ursolic acid, substituted benzaldehyde, and $o$-phenylendiamine or 2-aminothiophenol. The compounds were structurally confirmed by ${ }^{1} \mathrm{H}$ NMR, IR and HRMS techniques. The preliminary bioassay test demonstrated that compounds $\mathbf{4 d}$ and $\mathbf{4 g}$ had potent anti-inflammatory activity.
\end{abstract}

Keywords ursolic acid; benzimidazole; benzothiazole; anti-inflammatory

熊果酸即 $3 \beta$-羟基-熊果-12-烯-28-酸 $(3 \beta$-hydroxyurs-12-en-28-oic acid, UA, 1)属于 $\alpha$-香树脂醇 $(\alpha$-amyrin) 型五环三萜类化合物, 具有抗炎 ${ }^{[1,2]}$ 、抗癌 ${ }^{[3,4]}$ 、抗菌 ${ }^{[5]}$ 等生物活性, 且毒副作用较小. 但其脂溶性和水溶性均 比较差, 不易透过生物膜, 故与同类临床药物相比其药 效明显偏低 ${ }^{[6]}$. 鉴于此, 本文以熊果酸为先导物, 进行 结构修饰, 将具有良好抗炎、抗癌、抗菌等药理活 性 ${ }^{[7 \sim 10]}$ 的苯并咪(噻)唑杂环结构引入其母体, 合成了一 系列熊果酸苯并咪(噻)唑连苯酯衍生物 $4 \mathrm{a} \sim \mathbf{4 h}$, 期望通 过有效分子片段的引入来调整熊果酸的的亲水性等理 化特性, 从而改善或提高熊果酸的药理活性, 得到高活 性的新熊果酸衍生物.

本文以熊果酸为原料, 在三氟乙酸酩的活化作用下
与取代苯甲醛成酯后，再与邻苯二胺或邻氨基苯硫酚生 成熊果酸-2'-甲氧基-4'(5')-[2-苯并咪(噻)唑]-1'- 苯酯 $(\mathbf{4 a} \sim \mathbf{4 h})$, 合成路线见 Scheme 1.

\section{1 实验部分}

\section{1 仪器与试剂}

核磁共振(NMR): ${ }^{1} \mathrm{H}$ NMR (Varian INOVA-400), 以 $\mathrm{CDCl}_{3}, \mathrm{DMSO}-d_{6}$ 为溶剂, TMS 作内标; 高分辨质谱 (HRMS): TOF-MS (Waters Q-TOF-Premiter instrument); 红外光谱(IR): Perkin-Elmer 16PC-FT ( $\mathrm{KBr}$ 压片); 熔点 用 XI-4 显微熔点测定仪测定(温度计未经校正); 电子天 平：美国双杰兄弟有限公司，型号为 T-1000 型; 分析天 平: 北京赛多利斯天平有限公司, 型号为 BS210S. 薄层

*E-mail: chuandayouji217@163.com

Received April 1, 2011; revised August 3, 2011; accepted September 9, 2011. 


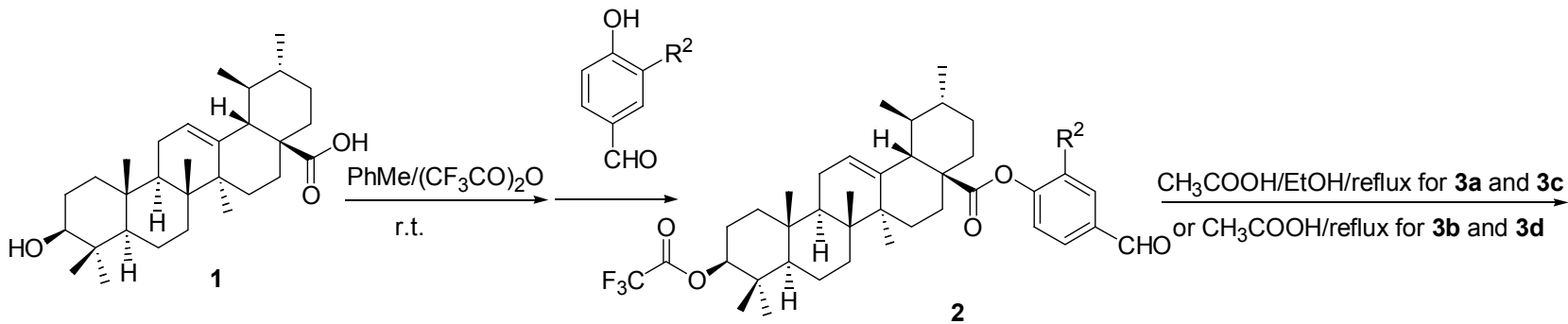

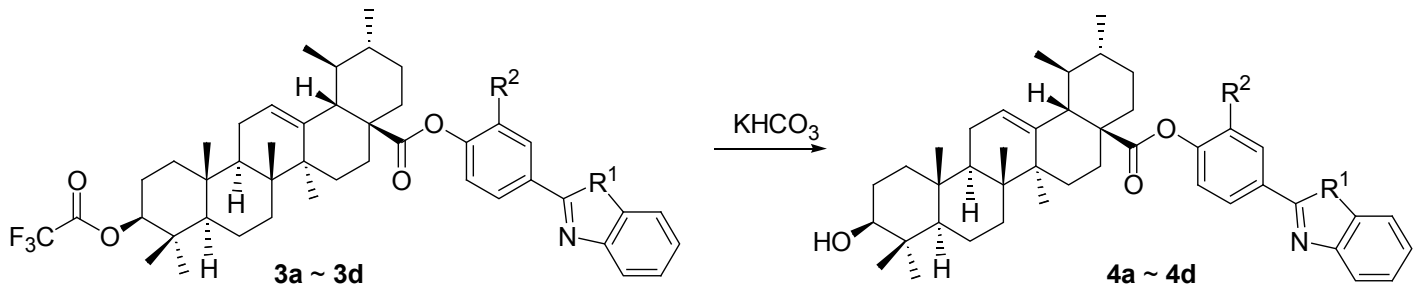

a: $R^{1}=N H, R^{2}=H ; \mathbf{b}: R^{1}=S, R^{2}=H ; c: R^{1}=N H, R^{2}=O_{3} ; \mathbf{d}: R^{1}=S, R^{2}=O_{3}$

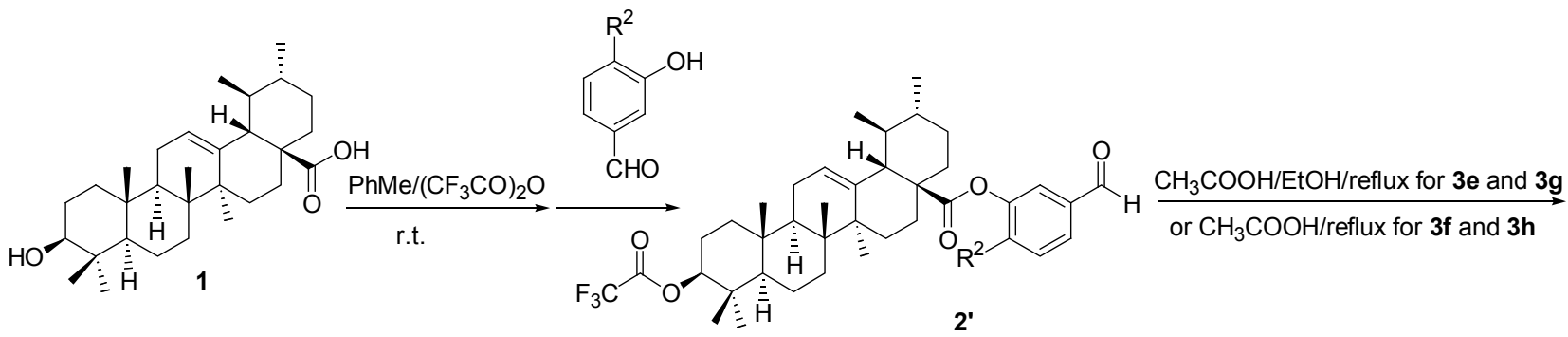

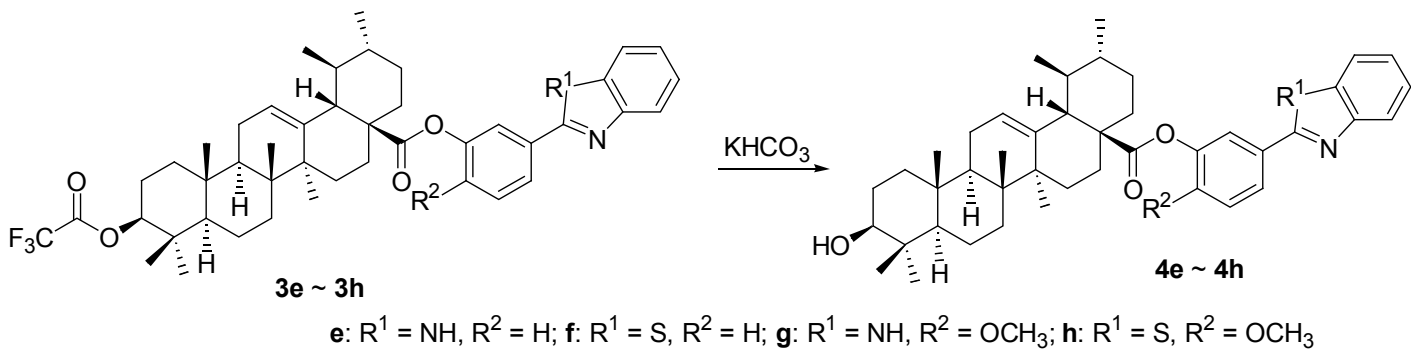

Scheme 1

板: 硅胶 $\mathrm{GF}_{254}$ (青岛海洋化工厂生产), $0.6 \%$ 的羧甲基纤 维素钠铺板, $110{ }^{\circ} \mathrm{C}$ 活化 $1 \mathrm{~h}$; 柱层析用 $300 \sim 400$ 目试 剂级硅胶(青岛海洋化工厂). 熊果酸: 西安江兴生物科 技有限公司提供, 纯度为 $95 \%$ 或以上; 所用试剂均为分 析纯或化学纯.

HepG2, A549 和 SKOV3 细胞: ATCC; DEME 培养 液: Gibco 公司(USA); 10\%牛血清: 兰州民海生物生物 工程有限公司. 动物: 昆明种小鼠, 体重 $18 \sim 23 \mathrm{~g}$, 䧳 雄各半, 由四川大学实验动物中心提供, 动物使用许可 证号: 第 10 号; 动物级别: 一级.

\section{2 合成}

1.2.13-三氟乙酰氧基熊果酸-2'-甲氧基-4'(5')-甲酰 基-1'-苯酯(2)和 3-三氟乙酰氧基熊果酸-4'(5')-甲酰基$1^{\prime}$-苯酯 $\left(2^{\prime}\right)$ 的合成

将 $2.28 \mathrm{~g}(5 \mathrm{mmol})$ 熊果酸溶于 $30 \mathrm{~mL}$ 甲苯中, 加入
$4.2 \mathrm{~mL}$ 三氟醋酸酎, 室温摚拌 $0.5 \mathrm{~h}$, 加入 $0.79 \mathrm{~g}(5.2$ $\mathrm{mmol}$ )香草醛, $80{ }^{\circ} \mathrm{C}$ 摚拌 $4 \mathrm{~h}$. 旋干溶剂后再加乙酸乙酯 溶解，用 $10 \% \mathrm{NaOH}$ 调至中性. 有机层用水、饱和 $\mathrm{NaCl}$ 溶液洗 2 3 次，用无水 $\mathrm{Na}_{2} \mathrm{SO}_{4}$ 干燥，过滤，浓缩. 再用 硅胶柱层析, 用 $V$ (石油醚) $: V($ 乙酸乙酯 $)=10 ： 1$ 洗脱, 得到白色絮状物 2, 同法可得 2'.

1.2.2 3-三氟乙酰氧基熊果酸-2'-甲氧基-4'(5')-(2-苯 并咪唑)-1'-苯酯 3a, 3c, 3e, 3g 的合成

将 $10 \mathrm{mmol}$ 熊果酸衍生物 2 溶解在 $5 \mathrm{~mL}$ 乙醇溶液 中, 并向此溶液中加入 $5 \mathrm{~d}$ 冰乙酸, 然后将此混合液缓 慢滴加到溶有 $10 \mathrm{mmol}$ 邻苯二胺的 $15 \mathrm{~mL}$ 乙醇溶液中, 进行回流反应，用 TLC 跟踪至反应完全，减压浓缩，用 $V$ (石油醚)： $V$ (丙酮 $)=5: 1$ 柱层析即得到 $\mathbf{3 a}$, 同法可得 $3 \mathbf{c}, 3 \mathbf{e}, 3 \mathrm{~g}$. 
1.2.3 3-三氟乙酰氧基熊果酸-2'-甲氧基-4'(5')-(2-苯 并噻唑)-1'-苯酯 3b, 3d, 3f, 3h 的合成

将 $10 \mathrm{mmol}$ 的邻氨基苯硫酚和熊果酸衍生物 2 依次 加入到 $10 \mathrm{~mL}$ 的乙酸溶液中, 然后加热回流, 用 TLC 跟 踪至反应完全, 混合物冷却至室温并倒入到冰水中, 析 出的固体过滤、干燥后用 $V$ (石油醚) $: V$ (丙酮 $)=5: 1$ 柱 层析，即得到 $\mathbf{3 b}$ ，同法可得 $\mathbf{3 d}, \mathbf{3 f}, \mathbf{3 h}$.

1.2.4 熊果酸-2'-甲氧基-4'(5')-[2-苯并咪(噻)唑]-1'-苯 酯 $(4 a \sim 4 h)$ 的合成

取 $50 \mathrm{mg}$ 的 $3 \mathrm{a}$ 纯品, 溶于 $5 \mathrm{~mL}$ 丙酮, 加入含 101 $\mathrm{mg}$ 的 $\mathrm{KHCO}_{3}$ 水溶液 $6 \mathrm{~mL}$, 室温搅拌反应 $1 \mathrm{~d}$, 反应完 全后, 用乙酸乙酯萃取 3 次, 有机层用无水 $\mathrm{Na}_{2} \mathrm{SO}_{4}$ 干燥, 过滤，减压浓缩得 $\mathbf{4 a}$, 同法可得 $\mathbf{4 b} \sim \mathbf{4 h}$.

4a: 白色粉末, 产率 $88 \%$. m.p. $310 \sim 312{ }^{\circ} \mathrm{C} ;{ }^{1} \mathrm{H}$ NMR (400 MHz, DMSO- $d_{6}$ ) $\delta$ : 13.04 (brs, 1H, NH), 8.20 (d, $J=8.52 \mathrm{~Hz}, 2 \mathrm{H}, \mathrm{ArH}), 7.60$ (s, 2H, ArH), $7.18 \sim 7.23$ (m, 4H, ArH), 5.27 (s, 1H, CH-12), 3.01 (brs, 1H, CH-3), 2.28 (d, $J=11.34 \mathrm{~Hz}, 1 \mathrm{H}, \mathrm{CH}-18), 1.24$ (s, 3H, $\left.\mathrm{CH}_{3}\right), 1.12$ (s, 3H, $\left.\mathrm{CH}_{3}\right), 0.91\left(\mathrm{~s}, 3 \mathrm{H}, \mathrm{CH}_{3}\right), 0.89$ (s, 3H, $\left.\mathrm{CH}_{3}\right), 0.86$ (d, $\left.J=6.80 \mathrm{~Hz}, 3 \mathrm{H}, \mathrm{CH}_{3}\right), 0.82\left(\mathrm{~s}, 3 \mathrm{H}, \mathrm{CH}_{3}\right), 0.68(\mathrm{~s}, 3 \mathrm{H}$, $\mathrm{CH}_{3}$ ); IR (KBr) v: 3202, 2924, 2868, 1749, 1618, 1497, 1451, 1379, 1274, 1208, 1166, 1134, 1092, 1028, 967, 859, $807,739 \mathrm{~cm}^{-1}$; HRMS (ESI) calcd for $\mathrm{C}_{43} \mathrm{H}_{57} \mathrm{~N}_{2} \mathrm{O}_{3}[\mathrm{M}+$ $\mathrm{H}]^{+}$649.4369, found 649.4376 .

4b: 白色粉末, 产率 52\%. m.p. 212 214 ${ }^{\circ} \mathrm{C} ;{ }^{1} \mathrm{H}$ NMR (400 MHz, $\left.\mathrm{CDCl}_{3}\right) \delta: 8.06 \sim 8.11(\mathrm{~m}, 3 \mathrm{H}, \mathrm{ArH})$, $7.90(\mathrm{~d}, J=8.00 \mathrm{~Hz}, 1 \mathrm{H}, \operatorname{ArH}), 7.50$ (t, $J=8.00 \mathrm{~Hz}, 1 \mathrm{H}$, $\operatorname{ArH}), 7.39$ (t, $J=7.60 \mathrm{~Hz}, 1 \mathrm{H}, \operatorname{ArH}), 7.16$ (d, $J=8.40 \mathrm{~Hz}$, 2H, ArH), 5.35 (s, 1H, CH-12), 3.23 (dd, $J=5.20,11.80$ $\mathrm{Hz}, 1 \mathrm{H}, \mathrm{CH}-3$ ), 2.32 (d, J=11.20 Hz, 1H, CH-18), 1.25 (s, $\left.3 \mathrm{H}, \mathrm{CH}_{3}\right), 1.15\left(\mathrm{~s}, 3 \mathrm{H}, \mathrm{CH}_{3}\right), 0.94\left(\mathrm{~s}, 3 \mathrm{H}, \mathrm{CH}_{3}\right), 0.90$ (d, $J=6.40 \mathrm{~Hz}, 3 \mathrm{H}), 0.88$ (s, 3H, $\left.\mathrm{CH}_{3}\right), 0.79$ (s, 3H, $\left.\mathrm{CH}_{3}\right)$; IR IR (KBr) v: 3440, 2926, 2862, 1751, 1606, 1482, 1456, 1381, 1205, 1162, 1132, 1092, 1029, 967, 857, 761, 728, $622 \mathrm{~cm}^{-1}$; HRMS (ESI) calcd for $\mathrm{C}_{43} \mathrm{H}_{56} \mathrm{NO}_{3} \mathrm{~S}[\mathrm{M}+\mathrm{H}]^{+}$ 666.3981, found 666.3937.

4c: 白色粉末, 产率 86\%. m.p. $305 \sim 307{ }^{\circ} \mathrm{C} ;{ }^{1} \mathrm{H}$ NMR (400 MHz, DMSO-d $) \delta$ : 12.92 (br, NH, 1H), 7.88 (d, $J=1.38 \mathrm{~Hz}, 1 \mathrm{H}, \mathrm{ArH}), 7.75$ (dd, $J=1.60,8.40 \mathrm{~Hz}, 1 \mathrm{H}$, ArH), $7.55 \sim 7.66(\mathrm{~m}, 2 \mathrm{H}, \operatorname{ArH}), 7.21(\mathrm{~d}, J=4.40 \mathrm{~Hz}, 2 \mathrm{H}$, ArH), 7.06 (d, J=8.40 Hz, 1H, ArH), 5.23 (s, 1H, CH-12), $3.86\left(\mathrm{~s}, 3 \mathrm{H}, \mathrm{OCH}_{3}\right), 3.01$ (brs, $\left.1 \mathrm{H}, \mathrm{CH}-3\right), 2.26$ (d, $J=$ $11.42 \mathrm{~Hz}, 1 \mathrm{H}, \mathrm{CH}-18), 1.11\left(\mathrm{~s}, 3 \mathrm{H}, \mathrm{CH}_{3}\right), 0.91$ (s, 3H, $\left.\mathrm{CH}_{3}\right), 0.89\left(\mathrm{~s}, 3 \mathrm{H}, \mathrm{CH}_{3}\right), 0.86\left(\mathrm{~d}, J=6.80 \mathrm{~Hz}, 2 \mathrm{H}, \mathrm{CH}_{3}\right)$, 0.83 (s, 3H, $\left.\mathrm{CH}_{3}\right), 0.69$ (s, 3H, $\mathrm{CH}_{3}$ ); IR (KBr) v: 3398,
2926, 2871, 1753, 1602, 1499, 1457, 1380, 1266, 1168, $1128,1033,1002,805,742 \mathrm{~cm}^{-1}$; HRMS (ESI) calcd for $\mathrm{C}_{44} \mathrm{H}_{59} \mathrm{~N}_{2} \mathrm{O}_{4}[\mathrm{M}+\mathrm{H}]^{+}$679.4475, found 679.4420.

4d: 白色粉末，产率 55\%. m.p. 239 241 ${ }^{\circ} \mathrm{C} ;{ }^{1} \mathrm{H}$ NMR (400 MHz, $\left.\mathrm{CDCl}_{3}\right) \delta: 8.06(\mathrm{~d}, J=8.40 \mathrm{~Hz}, 1 \mathrm{H}$, ArH), 7.89 (d, $J=8.00 \mathrm{~Hz}, 1 \mathrm{H}, \operatorname{ArH}), 7.78 \sim 7.79(\mathrm{~m}, 1 \mathrm{H}$, ArH), 7.57 (dd, $J=1.60,8.00 \mathrm{~Hz}, 1 \mathrm{H}, \mathrm{ArH}), 7.50$ (t, $J=$ $7.60 \mathrm{~Hz}, 1 \mathrm{H}, \mathrm{ArH}), 7.39$ (t, $J=7.60 \mathrm{~Hz}, 1 \mathrm{H}, \mathrm{ArH}), 7.04$ (d, $J=8.40 \mathrm{~Hz}, 1 \mathrm{H}, \mathrm{ArH}), 5.33$ (s, 1H, CH-12), 3.93 (s, 3H, $\left.\mathrm{OCH}_{3}\right), 3.23$ (dd, $\left.J=5.20,11.80 \mathrm{~Hz}, 1 \mathrm{H}, \mathrm{CH}-3\right), 2.38$ (d, $J=11.20 \mathrm{~Hz}, 1 \mathrm{H}, \mathrm{CH}-18), 1.15\left(\mathrm{~s}, 3 \mathrm{H}, \mathrm{CH}_{3}\right), 1.01$ (s, 3H, $\left.\mathrm{CH}_{3}\right), 0.95$ (s, 3H, $\left.\mathrm{CH}_{3}\right), 0.90\left(\mathrm{~s}, 3 \mathrm{H}, \mathrm{CH}_{3}\right), 0.89$ (d, J= $6.40 \mathrm{~Hz}, 3 \mathrm{H}), 0.80$ (s, 3H, $\mathrm{CH}_{3}$ ); IR (KBr) v: 3434, 2925, 2859, 1752, 1603, 1517, 1460, 1414, 1382, 1267, 1162, $1128,1093,1034,886,762,729,658 \mathrm{~cm}^{-1}$; HRMS (ESI) calcd for $\mathrm{C}_{44} \mathrm{H}_{58} \mathrm{NO}_{4} \mathrm{~S}[\mathrm{M}+\mathrm{H}]^{+}$696.4087, found 696.4004

4e: 白色粉末，产率 $85 \%$. m.p. 208 $210{ }^{\circ} \mathrm{C} ;{ }^{1} \mathrm{H}$ NMR (400 MHz, DMSO- $d_{6}$ ) $\delta: 12.99$ (br, 1H, NH), 8.04 (d, $J=7.65 \mathrm{~Hz}, 1 \mathrm{H}, \mathrm{ArH}), 7.86$ (s, 1H, ArH), 7.60 (t, $J=$ $8.00 \mathrm{~Hz}, 3 \mathrm{H}, \mathrm{ArH}), 7.23$ (s, 2H, ArH), 7.10 (d, J=8.00 Hz, 1H, ArH), 5.28 (s, 1H, CH-12), 3.01 (brs, 1H, CH-3), 2.30 (d, $J=11.42 \mathrm{~Hz}, 1 \mathrm{H}, \mathrm{CH}-18), 1.23$ (s, 3H, $\left.\mathrm{CH}_{3}\right), 1.11$ (s, $\left.3 \mathrm{H}, \mathrm{CH}_{3}\right), 0.90$ (s, 3H, $\left.\mathrm{CH}_{3}\right), 0.88$ (s, 3H, $\left.\mathrm{CH}_{3}\right), 0.86$ (d, $J=6.80 \mathrm{~Hz}, 3 \mathrm{H}), 0.82$ (s, 3H, $\left.\mathrm{CH}_{3}\right), 0.68\left(\mathrm{~s}, 3 \mathrm{H}, \mathrm{CH}_{3}\right)$; IR (KBr) v: 3391, 2925, 2855, 1750, 1616, 1586, 1540, 1455, 1379, 1268, 1188, 1135, 1096, 1031, 1001, 893, 741, 686 $\mathrm{cm}^{-1}$; HRMS (ESI) calcd for $\mathrm{C}_{43} \mathrm{H}_{57} \mathrm{~N}_{2} \mathrm{O}_{3}[\mathrm{M}+\mathrm{H}]^{+}$ 649.4369 , found 649.4380 .

4f: 白色粉末，产率 52\%, m.p. $122 \sim 124{ }^{\circ} \mathrm{C} ;{ }^{1} \mathrm{H}$ NMR (400 MHz, $\left.\mathrm{CDCl}_{3}\right) \delta: 8.07(\mathrm{~d}, J=8.00 \mathrm{~Hz}, 1 \mathrm{H}$, ArH), $7.91 \sim 7.93$ (m, 2H, ArH), 7.76 (s, 1H, ArH), 7.49 (q, $J=8.40 \mathrm{~Hz}, 2 \mathrm{H}, \mathrm{ArH}), 7.40$ (t, $J=7.60 \mathrm{~Hz}, 1 \mathrm{H}, \mathrm{ArH})$, $7.16 \sim 7.18$ (m, 1H, ArH), 5.37 (s, 1H, CH-12), 3.23 (dd, $J=5.20,11.80 \mathrm{~Hz}, 1 \mathrm{H}, \mathrm{CH}-3), 2.39$ (d, $J=11.20 \mathrm{~Hz}, 1 \mathrm{H}$, $\mathrm{CH}-18), 1.15$ (s, 3H, $\left.\mathrm{CH}_{3}\right), 0.96$ (s, 3H, $\left.\mathrm{CH}_{3}\right), 0.93$ (s, 3H, $\left.\mathrm{CH}_{3}\right), 0.91(\mathrm{~d}, J=6.80 \mathrm{~Hz}, 3 \mathrm{H}), 0.79$ (s, 3H, $\left.\mathrm{CH}_{3}\right)$; IR (KBr) v: 3442, 2925, 2868, 1748, 1586, 1509, 1442, 1381, 1311, 1216, 1163, 1112, 1091, 1030, 998, 890, 826, 759, $728,686 \mathrm{~cm}^{-1}$; HRMS (ESI) calcd for $\mathrm{C}_{43} \mathrm{H}_{56} \mathrm{NO}_{3} \mathrm{~S}[\mathrm{M}+$ $\mathrm{H}]^{+}$666.3981, found 666.3943 .

4g: 白色粉末，产率 $87 \%$. m.p. $245 \sim 247{ }^{\circ} \mathrm{C} ;{ }^{1} \mathrm{H}$ NMR (400 MHz, DMSO- $d_{6}$ ) $\delta: 12.94$ (br, 1H, NH), 8.16 $8.19(\mathrm{~m}, 1 \mathrm{H}, \mathrm{ArH}), 7.90 \sim 7.91(\mathrm{~m}, 1 \mathrm{H}, \mathrm{ArH}), 7.77$ (dd, $J=3.2,6.40 \mathrm{~Hz}, 2 \mathrm{H}, \mathrm{ArH}), 7.44 \sim 7.51$ (m, 3H, ArH), 5.23 
(s, 1H, CH-12), 3.37 (s, 3H, $\mathrm{OCH}_{3}$ ), 3.01 (brs, 1H, CH-3), $2.24(\mathrm{~d}, J=11.20 \mathrm{~Hz}, 1 \mathrm{H}, \mathrm{CH}-18), 1.11\left(\mathrm{~s}, 3 \mathrm{H}, \mathrm{CH}_{3}\right), 0.91$ (s, 3H, $\left.\mathrm{CH}_{3}\right), 0.88\left(\mathrm{~s}, 3 \mathrm{H}, \mathrm{CH}_{3}\right), 0.86(\mathrm{~d}, J=6.60 \mathrm{~Hz}, 2 \mathrm{H}$, $\left.\mathrm{CH}_{3}\right), 0.80\left(\mathrm{~s}, 3 \mathrm{H}, \mathrm{CH}_{3}\right), 0.67\left(\mathrm{~s}, 3 \mathrm{H}, \mathrm{CH}_{3}\right)$; IR (KBr) v: 3392, 2925, 2858, 1737, 1621, 1498, 1449, 1380, 1270, 1136, 1094, 1028, 813, $743 \mathrm{~cm}^{-1}$; HRMS (ESI) calcd for $\mathrm{C}_{44} \mathrm{H}_{59} \mathrm{~N}_{2} \mathrm{O}_{4}[\mathrm{M}+\mathrm{H}]^{+}$679.4475, found 679.4412 .

4h: 白色粉末, 产率 50\%. m.p. $148 \sim 150{ }^{\circ} \mathrm{C} ;{ }^{1} \mathrm{H}$ NMR (400 MHz, $\left.\mathrm{CDCl}_{3}\right) \delta: 8.02(\mathrm{~d}, J=8.00 \mathrm{~Hz}, 1 \mathrm{H}$, ArH), 7.93 (dd, $J=2.00,8.40 \mathrm{~Hz}, 1 \mathrm{H}, \operatorname{ArH}), 7.87$ (d, $J=$ $8.00 \mathrm{~Hz}, 1 \mathrm{H}, \mathrm{ArH}), 7.67$ (d, J=2.00 Hz, 1H, ArH), 7.48 (t, $J=7.60 \mathrm{~Hz}, 1 \mathrm{H}, \mathrm{ArH}), 7.36$ (t, $J=7.60 \mathrm{~Hz}, 1 \mathrm{H}, \mathrm{ArH}), 7.02$ (d, $J=8.40 \mathrm{~Hz}, 1 \mathrm{H}, \mathrm{ArH}), 5.36$ (s, 1H, CH-12), 3.86 (s, $\left.3 \mathrm{H}, \mathrm{OCH}_{3}\right), 3.23(\mathrm{dd}, J=5.20,11.80 \mathrm{~Hz}, 1 \mathrm{H}, \mathrm{CH}-3), 2.39$ (d, $J=11.20 \mathrm{~Hz}, 1 \mathrm{H}, \mathrm{CH}-18), 1.15$ (s, 3H, $\left.\mathrm{CH}_{3}\right), 1.01$ (s, $3 \mathrm{H}, \mathrm{CH}_{3}$ ), 0.97 (s, 3H, $\left.\mathrm{CH}_{3}\right), 0.95$ (s, 3H, $\mathrm{CH}_{3}$ ), 0.90 (d, $J=6.40 \mathrm{~Hz}, 3 \mathrm{H}), 0.79$ (s, 3H, $\left.\mathrm{CH}_{3}\right)$; IR (KBr) v: 3407, 2927, 2867, 1751, 1614, 1525, 1484, 1436, 1383, 1312, $1277,1215,1163,1129,1029,1001,899,807,756,725$, $696 \mathrm{~cm}^{-1}$; HRMS (ESI) calcd for $\mathrm{C}_{44} \mathrm{H}_{58} \mathrm{NO}_{4} \mathrm{~S}[\mathrm{M}+\mathrm{H}]^{+}$ 696.4087, found 696.4096.

\section{3 抗炎活性实验}

将化合物 $\mathbf{4 a} \sim \mathbf{4 h}$ 和 UA 用 $0.5 \%$ 羧甲基纤维素钠 $(\mathrm{CMC})$ 配制成浓度为 $7.5 \mathrm{mg} / \mathrm{mL}$ 混悬液, 阿司匹林片研 磨后用 $0.5 \% \mathrm{CMC}$ 配制成浓度为 $16.5 \mathrm{mg} / \mathrm{mL}$ 混悬液. 取 昆明种小鼠 66 只, 按体重随机分为 11 组, 每组 6 只, 䧳 雄各半. 即阴性对照组灌服( $0.5 \% \mathrm{CMC}$ 液) $0.4 \mathrm{~mL} / 20 \mathrm{~g}$; 阿司匹林组灌服(1.65\%阿司匹林) $0.4 \mathrm{~mL} / 20 \mathrm{~g}$; 各化合 物组分别灌服 $(0.75 \%$ 混悬液 $) 0.4 \mathrm{~mL} / 20 \mathrm{~g}$. 以上各组小 鼠, 每日给药 1 次, 连续给药 $4 \mathrm{~d}$, 于末次给药后 $0.5 \mathrm{~h}$,

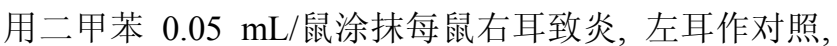
致炎后 $30 \mathrm{~min}$ 脱颈椎处死小鼠, 以 $9 \mathrm{~mm}$ 直径打孔器取 下耳缘左、右耳片, 分别用电子天平称重, 以右、左耳 片重量差表示肿胀度，进行组间显著差比较

\section{2 结果与讨论}

\section{1 合成}

熊果酸的结构中同时含有羧基和羟基, 其中 28 位 的䍨基空间位阻较大，较难发生酯化反应. 倘若采用活 性较高的酰氯法成酯, A 环 3 位羟基的影响不可忽略. 因此，本文采用在室温的条件下，将定量的熊果酸与三 氟乙酸酐直接进行反应，使其先转化为 3-三氟乙酰氧基 熊果酸三氟乙酸酐 ${ }^{[11,12]}$. 这样不仅可以保护 $\mathrm{A}$ 环 3 位的 差基, 而且还使熊果酸 28 位的羧基酰化能力得到增强,
易与取代苯甲醛成酯得 2 或 2 '. 该方法操作简便，反应 时间短，条件比较温和，是熊果酸与酚羟基化合物直接 进行酯化反应的一种较便利的方法.

在冰乙酸作催化剂的条件下，邻苯二胺和芳醛经缩 合、脱水环化、空气直接氧化可生成苯并咪唑环 ${ }^{[13]}$. 本 文以上述合成方法为依据，在冰乙酸催化下，成功地合 成了 3-三氟乙酰氧基熊果酸 2'-甲氧基-4'(5')-(2-苯并咪 唑)-1'-苯酯 3a, 3c, 3e, 3g. 为避免副产物双 Schiff 碱含量 的增多, 在操作过程中, 滴加速度一定要缓慢, 并严格 控制反应温度在 $5{ }^{\circ} \mathrm{C}$ 左右.

苯并噻唑环的合成 ${ }^{[14]}$ 是在乙酸作催化剂及溶剂的 条件下，直接对芳醛 2 或 2 与邻氨基苯硫酚的混合物进 行回流反应. 反应机理是邻氨基苯硫酚首先与芳醛形成 Schiff 中间体, 然后在加热的条件下会进一步环合生成 3-三氟乙酰氧基熊果酸 2'-甲氧基-4'(5')-(2-苯并噻唑)-1'苯酯 $(3 \mathbf{b}, 3 \mathbf{d}, \mathbf{3 f}, \mathbf{3 h})$.

由于熊果酸 28 位羧基的高位阻使其成酯后，较 $\mathrm{A}$ 环 3 位三氟乙酸酯难发生水解反应，因此，熊果酸-2'-甲 氧基-4'(5')-[2-苯并咪(噻)唑]-1'-苯酯 $(4 \mathbf{a} \sim 4 \mathbf{h})$ 的合成，则 是以 3-三氟乙酰氧基熊果酸衍生物 $3 \mathbf{a} \sim \mathbf{3 h}$ 为原料, 参 照文献的处理方法 ${ }^{[15]}$ ，以毒性较小的丙酮为溶剂，在 $\mathrm{KHCO}_{3}$ 的弱碱性条件下，将三氟乙酰基成功脱掉，得最 终目标产物 $\mathbf{4 a} \sim \mathbf{4 h}$.

\section{2 波谱解析}

\subsection{1 ${ }^{1} \mathrm{H}$ NMR 谱图}

所合成的化合物 $\mathbf{4 a} \sim \mathbf{4 h}$ 中，熊果酸 28 位羧基的质 子吸收峰消失, 熊果酸 C-12 的吸收峰出现在 $\delta 5.28$ 左 右; 熊果酸 C-18 的吸收峰出现在 $\delta 2.30$ 左右, 熊果酸 多个甲基的特征吸收峰出现在 $\delta \quad 0.67 \sim 1.25$ 范围内; 在 $\delta 7.02 \sim 8.20$ 范围内出现苯环的质子吸收峰, 其个数与 目标化合物苯环上的氢个数相符, 在 $\delta 12.94$ 左右是苯 并咪唑 $\mathrm{N}-\mathrm{H}$ 的质子吸收峰. 在化合物 $\mathbf{4 a} \sim \mathbf{4 h}$ 中, 熊果 酸母体环上的 $\mathrm{H}$ 的化学位移基本不变，说明连接苯并咪 唑和苯并噻唑对其母环影响不大.

\subsubsection{IR 谱图}

所合成的化合物 $\mathbf{4 a} \sim \mathbf{4 h}$ 中, $3440 \mathrm{~cm}^{-1}$ 附近出现宽 而强的 $\mathrm{O}-\mathrm{H}$ 伸缩振动吸收峰, $3202 \mathrm{~cm}^{-1}$ 附近出现了 $\mathrm{N}-\mathrm{H}$ 伸缩振动吸收峰, 一些化合物的 $\mathrm{N}-\mathrm{H}$ 伸缩振动 吸收峰与 $\mathrm{O}-\mathrm{H}$ 伸缩振动吸收峰重叠在一起; $2925 \mathrm{~cm}^{-1}$ 左右区域系甲基、亚甲基、次甲基碳氢键伸缩振动吸收 峰, 在化合物 $\mathbf{4 a} \sim \mathbf{4 h}$ 中, $1737 \sim 1752 \mathrm{~cm}^{-1}$ 范围内出现 $\mathrm{C}=\mathrm{O}$ 的吸收峰，在 $1602 \sim 1621 \mathrm{~cm}^{-1}$ 范围内出现 $\mathrm{C}=\mathrm{N}$ 的中等强度吸收峰，在 $1205 \sim 1277 \mathrm{~cm}^{-1}$ 范围内出现 $\mathrm{N}-\mathrm{C}=\mathrm{N}$ 和 $\mathrm{S}-\mathrm{C}=\mathrm{N}$ 的中等强度吸收峰. 


\subsubsection{MS 谱图}

目标化合物 $\mathbf{4 a} \sim \mathbf{4 h}$ 质子峰都以所预想的准分子离 子峰 $[\mathrm{M}+\mathrm{H}]^{+}$的形式出现, 说明该类化合物均比较稳定. HRMS 检测与目标化合物的分子组成完全一致.

\section{3 抗炎活性实验}

抗炎活性实验结果如表 1 所示. 与阴性对照组比较, 绝大部分目标化合物具有一定的抗炎活性. 其中, 4d $\left(150 \mathrm{mg} \cdot \mathrm{kg}^{-1}\right)$ 和 $4 \mathrm{~g}\left(150 \mathrm{mg} \cdot \mathrm{kg}^{-1}\right)$ 能明显减轻二甲苯致 小鼠耳廓炎症反应, 具有良好的抗炎活性. 与原料组熊 果酸比较, 目标化合物的抗炎活性有所减弱. 从目标化 合物的结构上看苯环上以甲氧基取代可能对抗炎活性 具有潜在的影响, 进一步深入研究正在进行中.

表 1 目标化合物的抗炎活性 ${ }^{a}$

Table 2 Anti-inflammatory activities for target compounds

\begin{tabular}{cccc}
\hline 组别 & 剂量 $/\left(\mathrm{mg} \cdot \mathrm{kg}^{-1}\right)$ & 动物数/只 & 肿胀度 $/(\mathrm{mg}, \bar{x} \pm \mathrm{SD})$ \\
\hline 阴性组 & - & 6 & $23.88 \pm 4.74$ \\
阿司匹林组 & 0.33 & 6 & $11.45 \pm 3.33^{* *}$ \\
$\mathbf{4 a}$ & 150 & 6 & $21.55 \pm 3.80$ \\
$\mathbf{4 b}$ & 150 & 6 & $20.51 \pm 6.73$ \\
$\mathbf{4 c}$ & 150 & 6 & $19.13 \pm 2.81$ \\
$\mathbf{4 d}$ & 150 & 6 & $16.87 \pm 5.35^{*}$ \\
$\mathbf{4 e}$ & 150 & 6 & $21.46 \pm 2.49$ \\
$\mathbf{4 f}$ & 150 & 6 & $20.88 \pm 2.31$ \\
$\mathbf{4 g}$ & 150 & 6 & $15.62 \pm 7.83^{*}$ \\
$\mathbf{4 h}$ & 150 & 6 & $19.88 \pm 6.11$ \\
$\mathrm{UA}$ & 150 & 6 & $14.21 \pm 5.48^{*}$ \\
\hline
\end{tabular}

${ }^{a}$ 与阴性组比: ${ }^{*} P<0.05, * * P<0.01$.

致谢 本论文的抗炎活性实验由四川大学华西药学院 药理教研室包旭老师等完成, 谱图数据由四川大学分析 测试中心提供, 在此表示衷心的感谢.

\section{References}

[1] (a) Baricevic, D.; Sosa, S.; Della, R.; Tubaro, A.; Simonovska, B.; Krosna, A. J. Ethnopharmacol. 2001, 75, 125. (b) Liu, J. J. Ethnopharmacol. 1995, 49, 57.

[2] Yang, D.-J.; Li, Y.; Yin, S.-F. Chin. J. Org. Chem. 2008, 28, 1055 (in Chinese). (杨定菊, 李顷, 尹述凡, 有机化学, 2008, 28, 1055.)

[3] Ma, C.-M.; Cai, S.-Q.; Cui, J.-R.; Wang, R.-Q.; Tu, P.-F.; Hattori, M. Eur. J. Med. Chem. 2005, 40, 582.

[4] Tatsuzaki, J.; Taniguchi, M.; Bastow, K. F.; Nakagawa-Goto, K.; Morris-Natschke, S. L.; Itokawa, H. Bioorg. Med. Chem. 2007, 15, 6193.

[5] Huang, M. T.; Ho, C. T.; Wang, Z. Y.; Ferraro, T.; Lou, Y. R.; Stauber, K. Cancer Res. 1994, 54, 701.

[6] Saraswat, B.; Vises, P. K.; Agarwa, D. P. Phytother. Res. 2000, 14, 163.

[7] Gromovaya, V. F.; Shapoval, G. S.; Luik, A. I.; Averkov, A. I.; Lozinskii, M. O. Russ. J. Gen. Chem. 1997, 67(6), 942.

[8] Craigo, W. A.; Lesueur, B. W.; Skibo, E. B. J. Med. Chem. 1999, 42(17), 3324.

[9] Wells, G.; Bradshaw, T. D.; Diana, P.; Seaton, A.; Shi, D.-F.; Westwell, A. D.; Stevens, M. F. G. Bioorg. Med. Chem. Lett. 2000, $10,513$.

[10] Rana, A.; Siddiqui, N.; Khan, S. A. Indian J. Pharm. Sci. 2007, 69, 10.

[11] Chen, L.; Zhang, Y.-H.; Bi, X.-L.; Luo, Y.-Q. Central South Pharm. 2006, 12, 416 (in Chinese). (陈莉, 奕华, 毕小玲, 罗叶青, 中南药学, 2006, 12, 416.)

[12] Parish, R. C.; Stock, L. M. Tetrahedron Lett. 1964, 5(20), 1285.

[13] Yang, H.-J.; Hu, C.; Li, Y.; Yin, S.-F. Chin. J. Org. Chem. 2008, 28,899 (in Chinese). (杨鸿均, 胡翠, 李颖, 尹述凡, 有机化学, 2008, 28, 899.)

[14] Kenny, R. S.; Mashelkar, U. C. J. Heterocycl. Chem. 2006, 43, 1367.

[15] Cramer, F.; Bär, H. P.; Rhaese, H. J.; Sänger, W.; Scheit, K. H.; Schneider, G.; Tennigkeit, J. Tetrahedron Lett. 1963, 4(16), 1039.

(Qin, X.; Li, L.) 